\title{
TIMP-4 and CD63: new prognostic biomarkers in human astrocytomas
}

Sandrine Rorive ${ }^{1}$, Xavier Moles Lopez ${ }^{1,2}$, Calliope Maris ${ }^{1}$, Anne-Laure Trepant ${ }^{1}$, Sébastien Sauvage ${ }^{1}$, Niloufar Sadeghi ${ }^{3}$, Isabelle Roland ${ }^{1}$, Christine Decaestecker ${ }^{2,4}$ and Isabelle Salmon ${ }^{1}$

${ }^{1}$ Department of Pathology, Erasme University Hospital, Brussels, Belgium; ${ }^{2}$ Laboratory of Image Synthesis
and Analysis (LISA), Faculty of Applied Sciences, Université Libre de Bruxelles, Brussels, Belgium and
${ }^{3}$ Department of Neuroimaging, Erasme University Hospital, Université Libre de Bruxelles, Brussels, Belgium

Based on the molecular profiling of astrocytomas, we previously identified a series of genes involved in astrocytoma invasion. Of these, tissue inhibitor of metalloproteinase-4 (TIMP-4) was found to be overexpressed in pilocytic astrocytomas relative to diffuse astrocytomas of any histological grade. Although some data suggest that TIMP-4 may be an anti-tumoral actor in astrocytomas, recent findings challenge this concept. The present study aims to investigate the diagnostic and prognostic values of TIMP-4 and its putative partner CD63 in human astrocytomas. Tissue microarray and image analysis were first carried out to quantitatively analyze the immunohistochemical expression of these proteins in 471 gliomas including 354 astrocytomas. Pathological semi-quantitative scores of both markers' expression were then established and correlated to astrocytoma diagnosis and patient prognosis. TIMP-4 and CD63 expressions were both overexpressed in astrocytomas compared with oligodendrogliomas $(P<0.001)$ and in pilocytic astrocytomas compared with grade II diffuse astrocytomas $(P<0.001)$. In glioblastomas, high TIMP-4/CD63 co-expression scores were identified as independent prognostic factors associated with progression and shorter survival. In conclusion, this work provides the first evidence of a TIMP-4/CD63 association in astrocytoma tumor cells. It identifies TIMP4 and CD63 as markers of the astrocytic phenotype in patients with gliomas. In addition, this work highlights the contribution of high TIMP-4/CD63 co-expression to the adverse outcomes of patients with glioblastomas. Modern Pathology (2010) 23, 1418-1428; doi:10.1038/modpathol.2010.136; published online 6 August 2010

Keywords: astrocytoma; CD63; diagnosis; image analysis; immunohistochemistry; marker; prognosis; TIMP

Invasive behavior is a pathological hallmark of diffuse gliomas that renders complete tumor resection impossible and leads to tumor recurrence and death of the patient. ${ }^{1}$ Diffuse gliomas include low-grade diffuse astrocytomas, anaplastic astrocytomas, glioblastomas, oligoastrocytomas and oligodendrogliomas; the latter two subgroups are subclassified into grades II and III (anaplastic). ${ }^{2}$ Among these, glioblastomas are associated with the worst prognosis, with most patients dying within 2 years after diagnosis. ${ }^{3}$ In marked contrast, surgical resection of generally noninfiltrative

Correspondence: Professor I Salmon, MD, PhD, Department of Pathology, Erasme University Hospital, 808 route de Lennik, Brussels B-1070, Belgium.

E-mail: Isabelle.Salmon@erasme.ulb.ac.be

${ }^{4}$ A Senior Research Associate with the 'Fond National de la Recherche Scientifique', Brussels, Belgium.

Received 18 February 2010; revised 14 June 2010; accepted 14 June 2010; published online 6 August 2010 pilocytic astrocytomas is usually associated with long-term survival or cure. ${ }^{2,4}$ Established prognostic factors such as histopathological type and grade, age, tumor location, multifocality, and extent of surgical resection are indicators of risk of recurrence after treatment. ${ }^{5}$ Recent advances in molecular biology, such as IDH1 mutation, BRAF gene duplication and concurrent deletion of chromosomal arms $1 \mathrm{p}$ and $19 \mathrm{q}$, provide new diagnostic and prognostic information regarding certain glioma subtypes. ${ }^{6-8}$ However, the overall outcomes for patients with diffuse gliomas remain unpredictable. ${ }^{2}$ New prognostic markers are still needed to identify high-risk patients (particularly in the setting of glioblastoma patients) for whom the standard treatment has poor outcomes and who would thus be well suited for more aggressive therapies. This clinical need motivated us to investigate the prognostic value of markers known to be involved in the invasion process of astrocytomas. 
Based on the molecular profiling of astrocytomas, we previously identified a series of genes involved in astrocytoma invasion. ${ }^{9}$ Of these, tissue inhibitor of metalloproteinase-4 (TIMP-4) was found to be overexpressed in pilocytic astrocytomas relative to all histological grades of diffuse astrocytomas. ${ }^{9}$ Tissue inhibitors of metalloproteinases are natural inhibitors of matrix metalloproteinases that are thought to regulate matrix metalloproteinase activity during extracellular matrix remodeling. ${ }^{10}$ This inhibitory activity might be important in preventing cancer invasion processes and malignant progression. ${ }^{11}$ TIMP-4 is widely expressed in the normal brain ${ }^{12}$ and was shown to reduce the invasive ability of glioblastoma cells in vitro. ${ }^{13}$ These data suggest that TIMP-4 may be an anti-tumoral factor in gliomas. However, this hypothesis has been challenged by some recent findings in other malignancies, such as breast, prostate, pancreatic and cervical cancers, wherein high TIMP-4 expression has been found ${ }^{14}$ to correlate with tumor aggressiveness ${ }^{15,16}$ and to stimulate tumorigenic activity. ${ }^{17}$ In addition, mounting evidence suggests an independent signaling capacity for tissue inhibitors of metalloproteinases distinct from their matrix metalloproteinase inhibitory activity. ${ }^{18}$ This theory is further supported by recent discoveries of tissue inhibitors of metalloproteinase-binding proteins. ${ }^{19-21}$ Among these, CD63 was shown to interact with TIMP-1 and TIMP-4. ${ }^{19,22}$ Although the TIMP-1/CD63 interaction was further confirmed and linked to an increase in cell survival signaling in human breast epithelial cells, the TIMP-4/ CD63 binding still remains hypothetical. CD63 is a tetraspanin that interacts with many different proteins, including integrins and the Src family tyrosine kinases Lyn and Hck; thus, it regulates intracellular signal transduction pathways involved in cell adhesion, motility and survival. ${ }^{23}$ To the best of our knowledge, data concerning CD63 expression in human gliomas are still missing. This paucity of information on CD63 and its putative link with TIMP-4, whose involvement in astrocytoma aggressiveness remains to be clarified, motivated us to evaluate the diagnostic and prognostic values of these two actors in human gliomas using quantitative immunohistochemistry based on image analysis of tissue microarray materials. ${ }^{24}$

\section{Materials and methods}

\section{Cell Culture and Cell Block Manufacturing}

Human LN229 high-grade astrocytoma cells (ATCC number CRL-2611) were maintained as previously described. ${ }^{25}$ For the preparation of cell blocks, subconfluent LN229 cells were washed twice with ice-cold PBS, detached with trypsin/EDTA (Invitrogen, Carlsbad, USA) and centrifuged at $500 \mathrm{~g}$ for $5 \mathrm{~min}$ at $4^{\circ} \mathrm{C}$. Pellets were submitted to Shandon_Cytoblock ${ }^{\mathbb{B}}$ reagents according to the manufacturer's recommendations (Thermo Fischer Scientific Inc., Waltham, USA).

\section{Immunoprecipitation}

Subconfluent LN229 cells were washed twice with ice-cold PBS and lysed at $4^{\circ} \mathrm{C}$ for $30 \mathrm{~min}$ with $1 \mathrm{ml}$ of lysis buffer (80 mM Tris-HCl, $150 \mathrm{mM} \mathrm{NaCl}, 1 \%$ Brij, $20 \mathrm{mM}$ EDTA, $4 \mathrm{mM} \mathrm{Na} \mathrm{VO}_{4}, 1 \mathrm{mM}$ PMSF and a protease inhibitor cocktail (Roche Diagnostics, Bale, Switzerland)), followed by $10 \mathrm{~min}$ of sonification. In all, $500 \mu \mathrm{g}$ of protein was immunoprecipitated overnight at room temperature with $2 \mu \mathrm{g}$ of anti-CD63 antibody (clone MX-49.129.5; Santa Cruz Biotechnology, Santa Cruz, CA, USA), followed by $2 \mathrm{~h}$ of incubation with $20 \mu \mathrm{l}$ of Protein A/G PLUS-Agarose (sc-2003, Santa Cruz Biotechnology). Controls used were water and normal mouse IgG (corresponding to the host species of the primary antibody). The immunoprecipitates were washed three times with ice-cold lysis buffer. The beads were resuspended in $30 \mu \mathrm{l}$ of Laemmli sample buffer, boiled for $3 \mathrm{~min}$ and centrifuged at $14000 \mathrm{~g}$ for $5 \mathrm{~min}$. The supernatants were fractionated by $12 \%$ SDS-PAGE under reducing conditions and submitted to immunoblotting with a goat polyclonal antibody against human TIMP-4 (C-16; sc-9375, Santa Cruz Biotechnology), as previously described. ${ }^{25}$ Each condition was run in three independent experiments.

\section{Patients and Tissue Samples}

Ten human glioma tissue microarrays were manufactured using archival formalin-fixed and paraffinembedded samples from 471 gliomas collected between 1984 and 2006 in the Department of Pathology of the Erasme Hospital, Brussels, Belgium. Three tissue cores per tumor (diameter: $600 \mu \mathrm{m}$ ), targeting the tumor bulk, were included in the tissue microarrays. For anaplastic astrocytomas and glioblastomas, three supplementary tissue cores, targeting the peritumoral zone, were added. The series includes 354 astrocytomas (42 grade I pilocytic astrocytomas, 26 grade II diffuse astrocytomas, 21 anaplastic astrocytomas and 265 glioblastomas), 91 oligodendrogliomas (62 grade II oligodendrogliomas and 29 anaplastic oligodendrogliomas) and 26 oligoastrocytomas (14 grade II oligoastrocytomas and 12 anaplastic oligoastrocytomas). All of these tumors are from patients who were not previously treated for brain tumors (primary tumors), and whose histopathological diagnoses were reviewed by two pathologists (SR and IS) to ensure consistent tumor grading based on guidelines from the 2007 revised WHO classification. ${ }^{2}$ The available clinical data for each patient included age, tumor site, multifocality and delineation, the extent of surgical resection, adjuvant treatment and followup (Table 1). Each tumor was classified as either well- or ill-circumscribed based on magnetic resonance imaging (retrospectively reviewed by a neuroradiologist (NS)) and/or surgical reports. Patient outcomes were characterized in terms of 
Table 1 Patient demographics and baseline features $(n=471)$

\begin{tabular}{|c|c|c|c|c|c|c|c|c|}
\hline & \multicolumn{4}{|c|}{ Astrocytomas $(\mathrm{n}=354)$} & \multicolumn{2}{|c|}{ Oligoastrocytomas $(\mathrm{n}=26)$} & \multicolumn{2}{|c|}{ Oligodendrogliomas $(\mathrm{n}=91)$} \\
\hline & $\begin{array}{l}\text { Grade I } \\
(\mathrm{n}=42)\end{array}$ & $\begin{array}{l}\text { Grade II } \\
(\mathrm{n}=26)\end{array}$ & $\begin{array}{c}\text { Grade III } \\
(\mathrm{n}=21)\end{array}$ & $\begin{array}{l}\text { Grade IV } \\
(\mathrm{n}=265)\end{array}$ & $\begin{array}{l}\text { Grade II } \\
(\mathrm{n}=14)\end{array}$ & $\begin{array}{c}\text { Grade III } \\
(\mathrm{n}=12)\end{array}$ & $\begin{array}{l}\text { Grade II } \\
(\mathrm{n}=62)\end{array}$ & $\begin{array}{l}\text { Grade III } \\
(\mathrm{n}=29)\end{array}$ \\
\hline \multicolumn{9}{|l|}{ Age (years) } \\
\hline$\leq 50$ & 39 & 21 & 17 & 39 & 11 & 5 & 51 & 17 \\
\hline$>50$ & 3 & 5 & 4 & 226 & 3 & 7 & 11 & 12 \\
\hline \multicolumn{9}{|l|}{ Multifocality } \\
\hline No & 40 & 25 & 20 & 202 & 14 & 11 & 62 & 27 \\
\hline Yes & 2 & 1 & 1 & 51 & & 1 & & 2 \\
\hline Missing & & & & 12 & & & & \\
\hline \multicolumn{9}{|l|}{ Delineation } \\
\hline Well-circumscribed & 26 & 1 & & & 2 & 1 & 5 & 3 \\
\hline Ill-circumscribed & 7 & 25 & 21 & 265 & 12 & 11 & 57 & 26 \\
\hline Missing & 9 & & & & & & & \\
\hline \multicolumn{9}{|l|}{ Corticosteroids } \\
\hline No & 25 & 14 & 4 & 39 & 7 & 3 & 43 & 11 \\
\hline Yes & 13 & 8 & 15 & 197 & 6 & 7 & 17 & 17 \\
\hline Missing & 4 & 4 & 2 & 29 & 1 & 2 & 2 & 1 \\
\hline \multicolumn{9}{|l|}{ Extent of surgery } \\
\hline Complete & 25 & 4 & 5 & 77 & 2 & 1 & 12 & 1 \\
\hline Partial & 14 & 22 & 16 & 168 & 12 & 10 & 47 & 28 \\
\hline Missing & 3 & & & 20 & & 1 & 3 & \\
\hline \multicolumn{9}{|l|}{ Adjuvant therapy } \\
\hline No & 40 & 18 & & 5 & 14 & 2 & 52 & 4 \\
\hline Radiotherapy & & 8 & 16 & 171 & & 4 & 3 & 7 \\
\hline Radiotherapy+temozolomide & & & 2 & 25 & & & & 1 \\
\hline Others $^{\mathrm{a}}$ & & & 3 & 18 & & 5 & 1 & 17 \\
\hline Missing & 2 & & & 46 & & 1 & 6 & \\
\hline \multicolumn{9}{|l|}{ Follow-up (months) } \\
\hline Range & $0-211$ & $2-288$ & $3-183$ & $0-48$ & $0-174$ & $0-140$ & 0-194 & $0-149$ \\
\hline Median & 73 & 70 & 28 & 8 & 59 & 19 & 57 & 32 \\
\hline Recurrence & $17.5 \%$ & $69.2 \%$ & $61.9 \%$ & $99.0 \%$ & $85.7 \%$ & $75.0 \%$ & $61.6 \%$ & $89.7 \%$ \\
\hline Death & $0 \%$ & $26.9 \%$ & $52.4 \%$ & $78.4 \%$ & $0 \%$ & $25.0 \%$ & $5.0 \%$ & $13.8 \%$ \\
\hline
\end{tabular}

The table displays the numbers (or percentages) of cases in the different glioma subtypes, except where other features are indicated (such as range and median).

${ }^{\text {a }}$ Including non-standard therapy for glioblastoma patients (grade IV astrocytomas), which are chemotherapy alone or combined with radiotherapy or palliative management).

disease-free survival and cancer-specific survival. The recurrences were defined as cases presenting magnetic resonance imaging evidence of progression that required a second surgery or adjuvant treatments. Disease-free survival and cancer-specific survival were measured from the date of tumor surgery until the date of recurrence or death (disease-free survival) or the date of death due to tumor progression (cancer-specific survival). In addition, two normal brain tissue microarrays were manufactured from 10 human post mortem brains (patients without neuropathological symptoms) obtained within $24 \mathrm{~h}$ of death. A minimum of six cores per specific region (diameter: $600 \mu \mathrm{m}$ ) targeted the cerebral hemisphere, the cerebellum, the brainstem and the cervical spinal cord.

\section{Immunohistochemistry}

As previously described, ${ }^{26}$ standard immunohistochemistry was applied to single 5 - $\mu$ m-thick sections cut from tissue microarrays and the LN229 cell block to display TIMP-4 or CD63 expression using rabbit polyclonal anti-TIMP-4 (1:100; AB19168; Chemicon Int., Temecula, CA, USA) and mouse monoclonal anti-CD63 (1:100; clone MX-49.129.5; Santa Cruz Biotechnology) antibodies. Negative controls were obtained by replacing the primary antibodies with non-immune serum (Dako, Glostrup, Denmark). In addition, technical and fixative controls were prepared on tissue microarray slides using hematoxylin-eosin and anti-vimentin staining, respectively. The control slides were checked using Spot 
Browser $^{\circledR}$ V2e (Alphelys, Plaisir, France) connected to a DXC-390 Sony camera and a motorized stage (Marzhaüser, Wetzlar-Steindorf, Germany) on a BX50 Olympus microscope (Aartselaar, Belgium). The final validation stage (conducted by a pathologist (SR)) aimed to confirm the adequacy of the specific tumor zones targeted and immunostaining compliance. Only the cores satisfying all the control steps were submitted for quantification. ${ }^{24}$ The tissue microarray core image acquisition and staining quantification were performed using Spot Browser ${ }^{\mathbb{R}}$ V2e, as recently described. ${ }^{24}$ For each valid core, we measured the analyzed (ie, positive and negative) tissue area and the positive (ie, stained) area. To reduce contamination of positive normal cells in tumor samples, only the tumor bulk was analyzed for each tumor, except for diffuse grade II astrocytomas, wherein there is generally no tumor bulk. In these cases, only the cores that exhibited hypercellularity and cellular atypia were taken into account for the analysis. To characterize each glioma or each specific brain region under study, we pooled all the concerned cores and computed the labeling index (LI), which is the percentage of the immunostained tissue area. To enable pathological specific applications, the value distributions of the TIMP-4 LI and CD63 LI were analyzed and scored using a three-tier semi-quantitative system: low, 0-10\%; moderate, 10-25\%; high, $>25 \%$.

\section{Statistical Analyses}

The comparison of proportions was carried out using the exact Fisher test $(2 \times 2$ cases $)$. The Kruskal-Wallis test was used to compare independent groups of numerical data. When this multi-group test was significant, post-hoc tests (the Dunn procedure) were used to compare the group pairs of interest, thus avoiding multiple comparison effects. Survival data were analyzed using the standard Kaplan-Meier analysis and the multivariate Cox regression. Survival curves were compared using the log-rank test. All the statistical analyses were carried out using Statistica (Statsoft, Tulsa, OK, USA).

\section{Results}

\section{TIMP-4 and CD63 are Differentially Expressed in Gliomas}

The baseline characteristics of the 471 patients with newly diagnosed gliomas are described in Table 1. The immunohistochemical staining of TIMP-4 and CD63 in some glioma subtypes is illustrated in Figure 1 and quantitative staining evaluations are shown in Figure 2. High TIMP-4 LI was detected in all normal brain samples (Figure 2a). When focusing on the different localizations analyzed, a weak predilection was observed for cerebellar samples compared with cerebral ones $(P<0.05$; data not shown). Neurons, astrocytes, endothelial cells and microglia express TIMP-4 in the cytoplasm. In some neurons and astrocytes we also observed a nuclear TIMP-4 staining (data not shown). TIMP-4 expression is reduced in astrocytomas as compared with normal brain samples $(P<0.01$, Figure 2a) and its expression is particularly low in oligodendrogliomas and oligoastrocytomas (Figure 2a). Normal oligodendrocytes as well as neoplastic cells with oligodendroglial differentiation lack TIMP-4 expression. In astrocytomas, TIMP-4 localizes to tumor cells but is rarely expressed in endothelial cells. In tumor astrocytes, we observed a TIMP-4 cytoplasmic and nuclear staining (Figure 1).

As opposed to TIMP-4, CD63 is weakly expressed in normal brain (Figure 2b), but its expression is significantly greater in astrocytomas compared with normal brain $(P<0.01$; Figure $2 \mathrm{~b})$ or with both oligodendrogliomas and oligoastrocytomas $(P<0.001$; Figure 2b). CD63 localizes mainly to astrocytoma tumor cells and to endothelial cells but is absent in oligodendroglial tumor cells. CD63 demonstrates a granular appearance in the cytoplasm of tumor astrocytes and of endothelial cells, which is sometimes associated with irregular membranous staining without any nuclear staining (Figure 1). In oligodendrogliomas and oligoastrocytomas, minigemistocytes and reactive astrocytes express both TIMP-4 and CD63 (see the arrows in Figure $1 \mathrm{~g}$ and $\mathrm{h}$ ).

When focusing on glioma type, we noted similarities in the distribution of TIMP-4 and CD63 expression (Figure 2a and b). In astrocytomas, both markers were dramatically overexpressed in pilocytic astrocytomas as compared to in diffuse grade II astrocytomas $(P<0.001)$, and both were elevated in anaplastic astrocytomas and/or glioblastomas as compared to in diffuse grade II astrocytomas (Figure $2 \mathrm{a}$ and $\mathrm{b}$ ). In addition, TIMP-4 and CD63 were both weakly expressed in grade II and grade III oligodendrogliomas (Figure 2a and b). Finally, CD63 expression significantly increased with tumor grade in oligoastrocytomas ( $P<0.05$; Figure $2 \mathrm{~b})$, whereas the slight increase in TIMP-4 expression observed in anaplastic oligoastrocytomas was not significant (Figure 2a). In glioblastomas, both TIMP-4 and CD63 expression were significantly reduced in the invasive part of the tumor compared with the tumor bulk $\left(P=0.01\right.$ and $P<10^{-6}$, respectively; data not shown).

\section{CD63 Could Help to Distinguish Pilocytic Astrocytomas from Grade II Diffuse Astrocytomas}

For clinical purposes, semi-quantitative scoring systems are routinely applied in pathology. ${ }^{27,28}$ In view of the expression data described above, we evaluated the putative diagnostic value of a three-tier scoring system (see Materials and 
TIMP-4
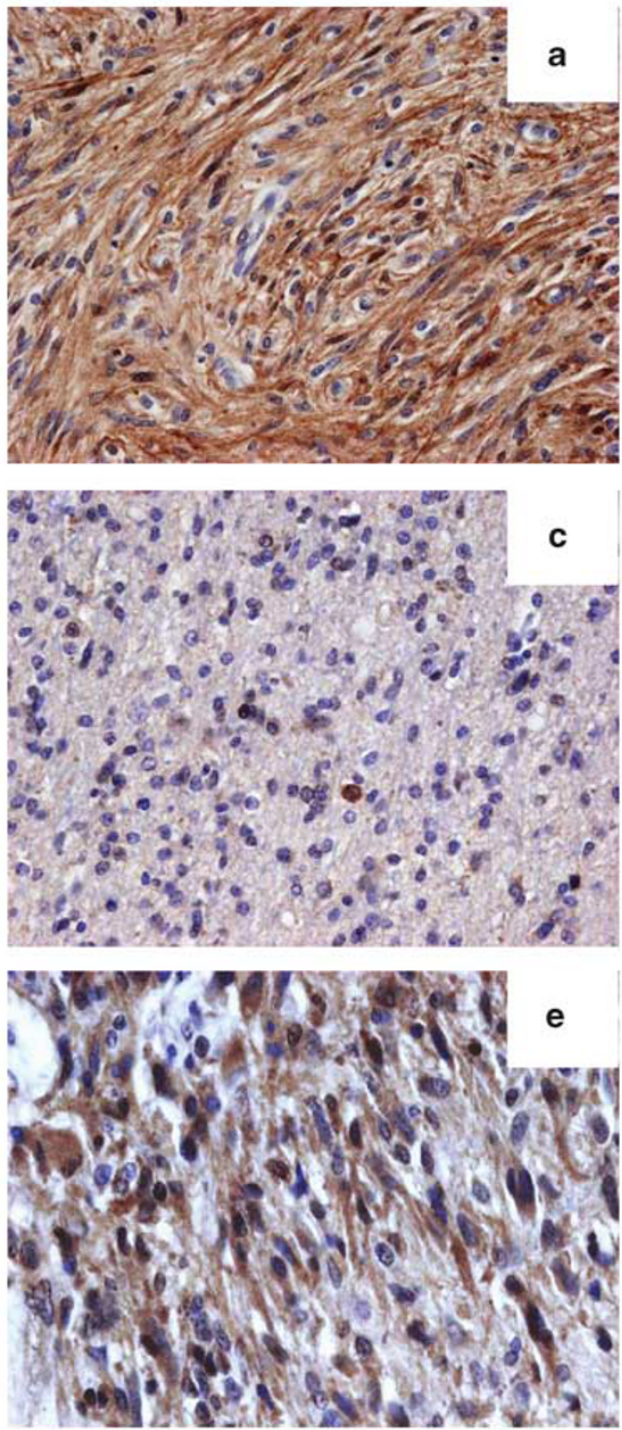

GLIOBLASTOMA

ANAPLASTIC OLIGODENDROGLIOMA
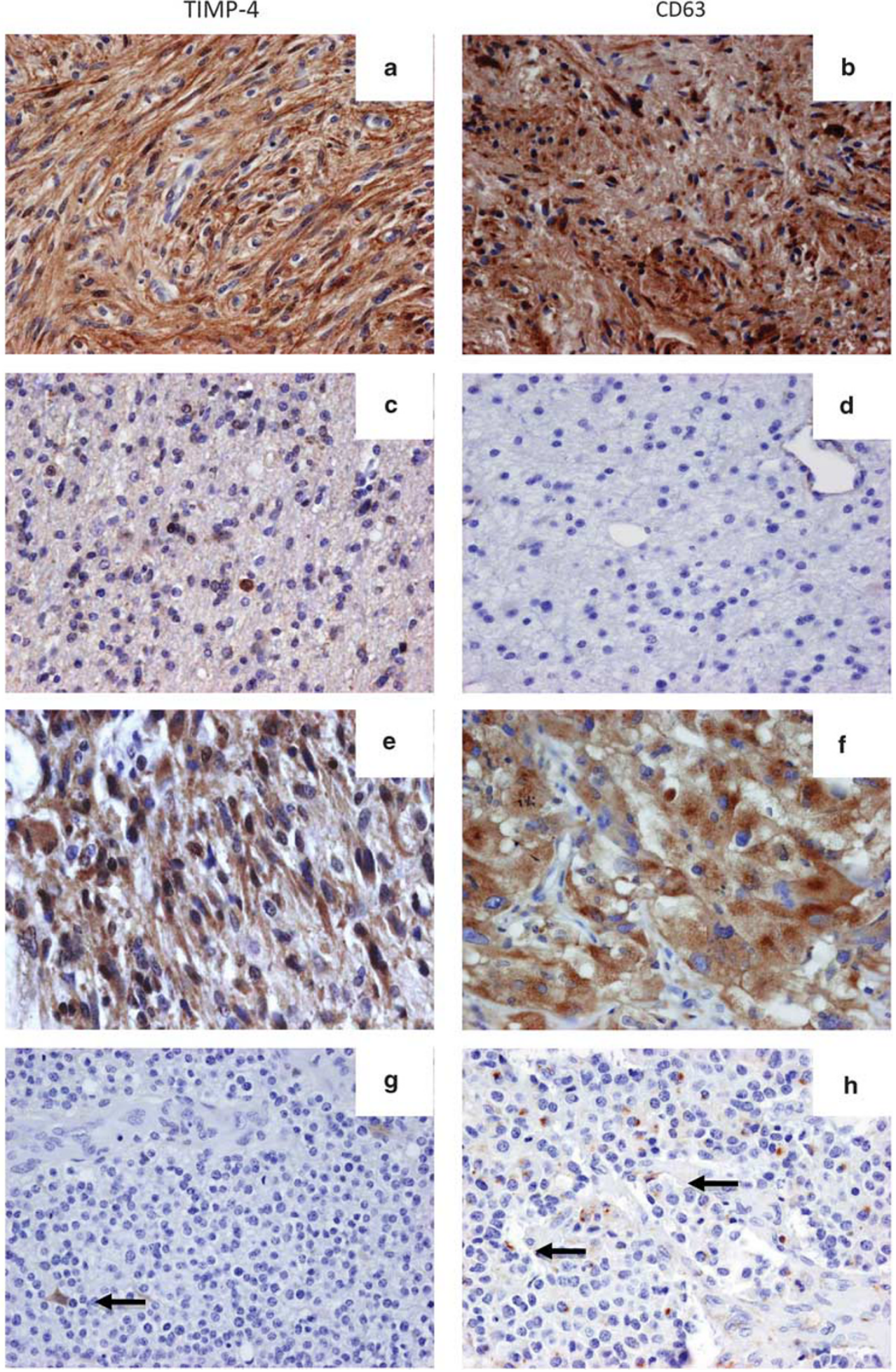

Figure 1 Immunohistochemical expression of TIMP-4 and CD63 in grade I pilocytic astrocytoma (a, b: $\times 400)$, grade II diffuse astrocytoma $(\mathbf{c}, \mathbf{d}: \times 400)$, glioblastoma $(\mathbf{e}, \mathbf{f}: \times 400)$ and anaplastic oligodendroglioma $(\mathbf{g}, \mathbf{h}: \times 400)$. Arrows illustrate microgemistocytes and reactive astrocytes' staining.

methods). We analyzed the sensitivity, specificity, positive and negative predictive values, efficiency and Fisher test $P$-value of the TIMP-4 LI and CD63 LI threshold values from the scoring system in order to identify the pathological semi-quantitative score that best discriminates between grade II diffuse astrocytomas and pilocytic astrocytomas. As shown in Figure 3, of the 354 astrocytomas analyzed, the best scoring, which consists of a comparison of CD63 $\mathrm{LI} \leq 10 \%$ vs a $\mathrm{CD} 63 \mathrm{LI}>10 \%$, separates 

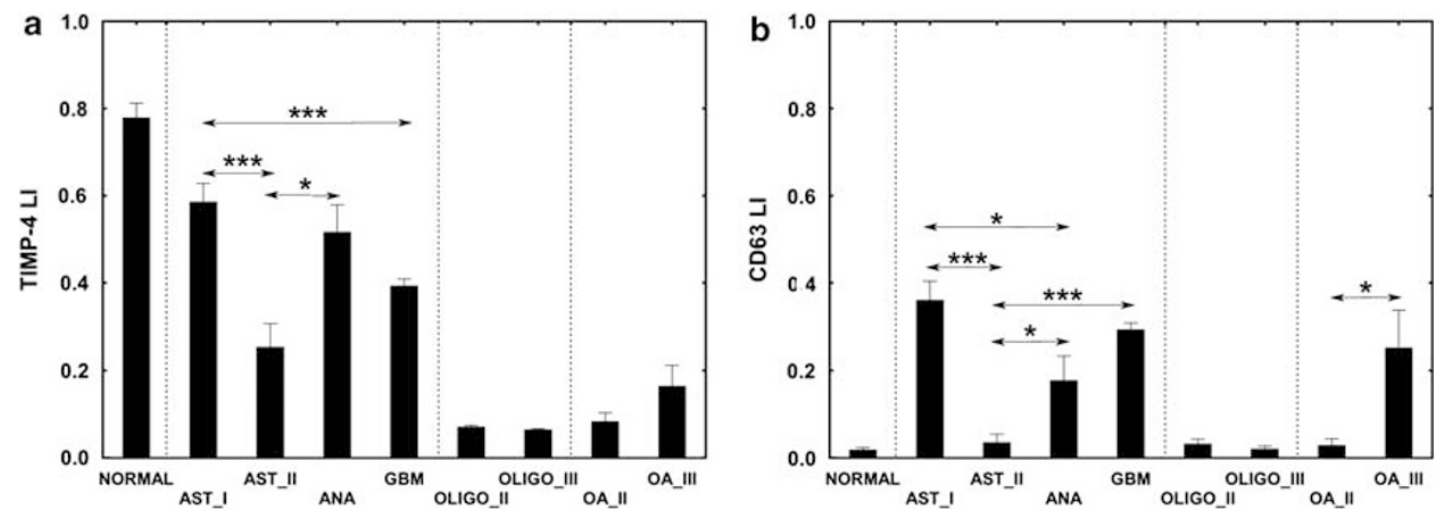

Figure 2 Quantitative evaluation of the percentage of tissue area exhibiting TIMP-4 (a) or CD63 (b) immunopositivity (LI, labeling index) in 10 normal brains (all regions taken into account; NORMAL) and in 471 gliomas with respect to histopathological type and grade. AST_I = pilocytic astrocytomas $(n=42)$; AST_II=grade II diffuse astrocytomas $(n=26)$; ANA=anaplastic astrocytomas $(n=21)$; GBM = glioblastomas $(n=265)$; OLIGO_II = grade II oligodendrogliomas $(n=62)$; OLIGO_III = anaplastic oligodendrogliomas $(n=29)$; OA_II = grade II oligoastrocytomas $(n=14)$; OA_III = anaplastic oligoastrocytomas $(n=12)$. Data are expressed in terms of means \pm s.e. Only the significant differences (post hoc multi-comparison tests) in each glioma subtypes are indicated, as ${ }^{*} P<0.05$ and ${ }^{* * *} P<0.001$.

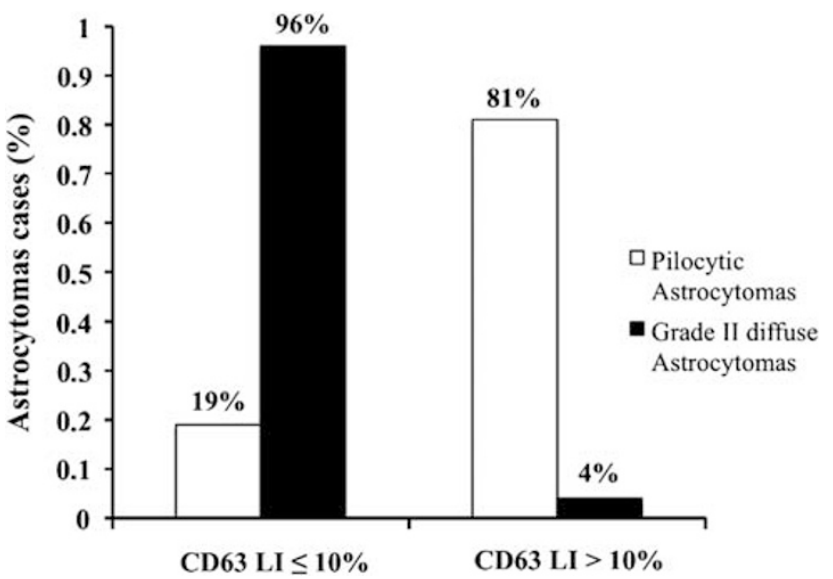

Figure 3 Illustration of the semi-quantitative score (expressed in terms of percentage of CD63 LI) identified to discriminate at best between pilocytic astrocytomas $(n=42)$ and diffuse grade II astrocytomas $(n=26)$. Of the 354 astrocytomas analyzed, such a scoring (which consists in a comparison of CD63 $\mathrm{LI} \leq 10 \%$ vs CD63 LI $>10 \%$ ) separates grade II diffuse astrocytomas from pilocytic astrocytomas with a specificity of $81 \%$ and a sensitivity of $96 \%\left(P<10^{-6}\right)$

grade II diffuse astrocytomas from pilocytic astrocytomas with a specificity of $81 \%$, a sensitivity of $96 \%$, a positive predictive value of $76 \%$, a negative predictive value of $97 \%$ and an efficiency of $87 \%$ $\left(P<10^{-6}\right)$.

\section{TIMP-4 and CD63 are Prognostic Markers in Glioblastomas, But Not in Pilocytic Astrocytomas}

Pilocytic astrocytomas and glioblastomas both exhibited high expression of TIMP-4 and CD63 despite the big biological differences between these two astrocytoma types (Figures 1 and 2). We therefore postulated that the biological implications and, consequently, the prognostic values of TIMP-4 and CD63 should be different in both tumor entities. To evaluate the prognostic contributions of TIMP-4 and CD63 in patients with astrocytomas, we first analyzed the impact of the clinical factors (listed in Table 1) on disease-free survival and cancerspecific survival. Considering the strong impact of histological grade on astrocytoma patient survival (data not shown), the analyses were carried out per grade. Univariate survival analyses revealed that none of the clinical factors influenced patient outcomes with either grade II diffuse astrocytomas or anaplastic astrocytomas in our series. In contrast, partial surgery was associated with a higher rate of tumor recurrence for patients with pilocytic astrocytomas $(P=0.02)$ (data not shown). The significant effects observed in the glioblastoma patients are detailed in Table 2. Older age was associated with reduced median survival for patients with glioblastoma $(P=0.0003)$. As expected, a macroscopically complete surgery significantly improved the median survival of these patients (from 8.2 to 12.7 months), as did the addition of temozolomide to radiotherapy (from 10.2 to 14.2 months $) \quad(P=0.0003$ and $P=0.008)$.

With regard to TIMP-4 and CD63 expressions, we did not find any significant impact on the outcome of patients with pilocytic astrocytoma (data not shown). In marked contrast, in glioblastoma patients, TIMP-4 LI, CD63 LI and TIMP-4/CD63 co-expressions (ie, TIMP-4 LI $\times$ CD63 LI) were all negatively associated with disease-free survival $(P=0.005, \quad P=0.01$ and $P=0.007$, respectively; Table 2). Moreover, high TIMP-4/CD63 co-expression was significantly associated with reduced survival in glioblastoma patients $(P=0.003$; Table 2 ). To enable clinical daily application, we analyzed the different TIMP-4 LI and CD63 LI threshold values from the scoring system (detailed in 
Table 2 Univariate survival analyses for patients with glioblastoma

\begin{tabular}{|c|c|c|c|c|}
\hline & $\begin{array}{c}\text { Median disease-free survival, } \\
\text { months ( } \pm \text { s.e.) }\end{array}$ & $\mathrm{P}$-value & $\begin{array}{l}\text { Median cancer-specific } \\
\text { survival, months ( } \pm \text { s.e.) }\end{array}$ & $\mathrm{P}$-value \\
\hline Age (years)* & $4.8 \pm 0.03$ & 0.05 & $9.1 \pm 0.03$ & 0.0003 \\
\hline Multifocality & & $>0.1$ & & 0.05 \\
\hline No & $4.8 \pm 0.04$ & & $9.9 \pm 0.04$ & \\
\hline Yes & $3.5 \pm 0.07$ & & $7.6 \pm 0.07$ & \\
\hline Corticosteroids & & $>0.1$ & & $>0.1$ \\
\hline No & $5.2 \pm 0.08$ & & $10.4 \pm 0.08$ & \\
\hline Yes & $4.4 \pm 0.04$ & & $8.7 \pm 0.04$ & \\
\hline Extent of surgery & & 0.0008 & & 0.0003 \\
\hline Partial & $4.0 \pm 0.04$ & & $8.2 \pm 0.04$ & \\
\hline Complete & $5.7 \pm 0.06$ & & $12.7 \pm 0.06$ & \\
\hline Adjuvant therapy & & 0.03 & & 0.008 \\
\hline Radiotherapy & $4.8 \pm 0.04$ & & $10.2 \pm 0.04$ & \\
\hline Radiotherapy+temozolomide & $7.2 \pm 0.1$ & & $14.2 \pm 0.1$ & \\
\hline TIMP-4 LI* & $4.8 \pm 0.03$ & 0.005 & $9.1 \pm 0.03$ & 0.1 \\
\hline CD63 LI* & $4.8 \pm 0.03$ & 0.01 & $9.1 \pm 0.03$ & 0.05 \\
\hline TIMP-4 LI × CD63 LI* & $4.8 \pm 0.03$ & 0.007 & $9.1 \pm 0.03$ & 0.003 \\
\hline Pathological application (scores) & & 0.02 & & 0.01 \\
\hline TIMP-4 $\mathrm{LI} \leq 10 \%$ or $\mathrm{CD} 63 \mathrm{LI} \leq 10 \%$ & $5.8 \pm 0.05$ & & $10.3 \pm 0.05$ & \\
\hline \multirow[t]{2}{*}{ TIMP-4 LI >10\% and CD63 LI>10\% } & $4.0 \pm 0.04$ & & $8.3 \pm 0.04$ & \\
\hline & & 0.06 & & 0.01 \\
\hline TIMP-4 $\mathrm{LI} \leq 25 \%$ or $\mathrm{CD} 63 \mathrm{LI} \leq 25 \%$ & $5.2 \pm 0.05$ & & $9.9 \pm 0.04$ & \\
\hline TIMP-4 LI $>25 \%$ and CD63 LI $>25 \%$ & $3.8 \pm 0.06$ & & $7.5 \pm 0.06$ & \\
\hline
\end{tabular}

Variables are considered as continuous variables in univariate Cox regression (see *) or two-class factors analyzed by the log-rank test. Each category is characterized by the median disease-free survival and the median cancer-specific survival time (in months) and their standard error (s.e.).

Materials and methods) in order to characterize at best the negative prognostic impact of these markers on disease-free survival and cancer-specific survival. As shown in Table 2 and Figure $4 \mathrm{a}$ and b, a score corresponding to 'TIMP-4 LI $>10 \%$ associated with CD63 LI>10\%' significantly decreases disease-free survival and cancer-specific survival in patients with glioblastoma. The negative prognostic impact of a high TIMP-4/CD63 co-expression (TIMP$4 \mathrm{LI}>25 \%$ and CD63 LI $>25 \%$ ) was particularly relevant for the group of glioblastoma patients who had macroscopically complete resections (Figure 4c and d). In this group, the median survival decreased to 9.1 months for glioblastomas exhibiting high TIMP-4/CD63 co-expression, as compared with 15.4 months for glioblastomas with lower co-expression $(P=0.0009$, Figure $4 \mathrm{~d})$. This latter result suggests that high TIMP-4/CD63 co-expression could be an independent prognostic factor regarding the extent of surgery for glioblastoma patients. To confirm this property, we performed a multivariate survival analysis by combining TIMP-4/CD63 co-expression with the clinical factors for which the univariate results in Table 2 indicated a $P$-value $<0.1$. Table 3 shows the two final models from which 'age' or 'multifocality' was excluded because of the $P$-value being $>0.1$ in the respective complete multivariate model. These final models highlighted a high TIMP4/CD63 co-expression pattern as being an indepen- dent prognostic factor associated with progression and worse survival for glioblastoma patients $(P=0.04$ and $P=0.02)$.

\section{TIMP-4 Interacts with CD63 in LN229 Astrocytoma Cells}

Finally, we studied the putative binding between TIMP-4 and CD63 in astrocytoma cells by carrying out in vitro immunoprecipitation experiments using the human LN229 glioblastoma cell line. As illustrated in Figure 5a and b, LN229 cells express both CD63 and TIMP-4 proteins. We showed that the anti-CD63 antibody co-immunoprecipitated endogenous CD63 with endogenous TIMP-4 (Figure 5, lane 3), whereas the specific mouse isotype had no effect (Figure 5, lane 2).

\section{Discussion}

Tumor recurrence and progression is common for gliomas; thus, continued research efforts are necessary to improve clinical treatments and outcomes. Although previous studies have identified several clinical and molecular factors that help to explain the variability in the outcomes of patients with gliomas, ${ }^{5}$ new markers are needed to more accurately recognize the high-risk patients, 

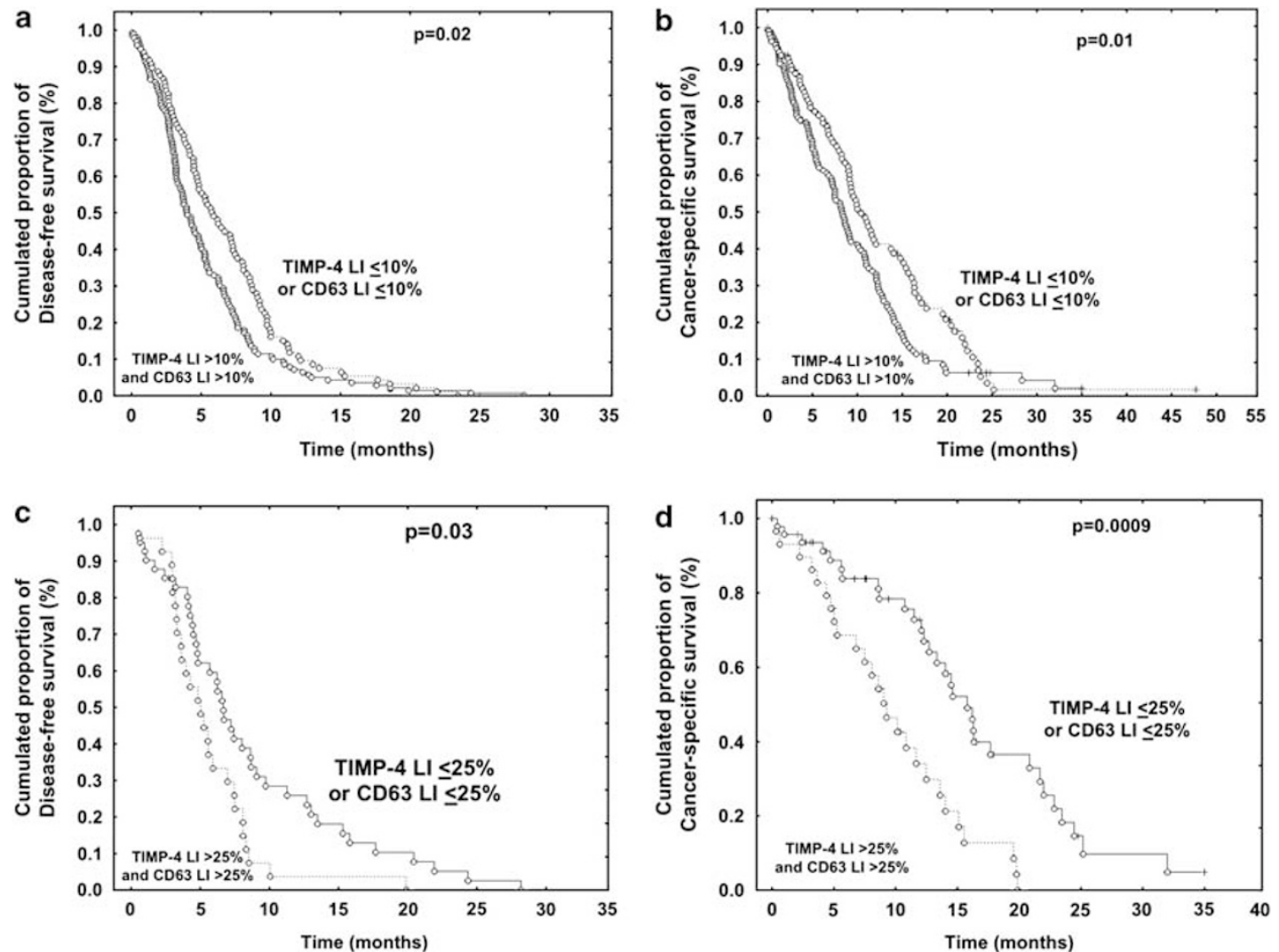

Figure 4 Kaplan-Meier curves characterizing (a-c) disease-free survival and (b-d) cancer-specific survival for glioblastoma patients ((a, b) all cases $(n=265)$, $(\mathbf{c}, \mathbf{d})$ homogeneous population of post-macroscopically complete surgery glioblastoma patients $(n=77))$, according to TIMP-4/CD63 co-expression scores. Scores: TIMP-4 LI>10\% associated with CD63 LI>10\% vs all the other cases (a, b), and TIMP-4 $\mathrm{LI}>25 \%$ associated with CD63 $\mathrm{LI}>25 \%$ vs all the other cases $(\mathbf{c}, \mathbf{d})$.

Table 3 Cox proportional hazards analyses of prognostic factors for patients with glioblastoma

\begin{tabular}{|c|c|c|c|c|c|}
\hline Glioblastoma $(\mathrm{n}=265)$ & Model/P-value & Prognostic factors & $\beta$ & $e^{\beta}$ & $\mathrm{P}$-value \\
\hline Disease-free survival & $P=0.0004$ & $\begin{array}{l}\text { Complete surgery } \\
\text { Radiotherapy+temozolomide } \\
\text { TIMP-4 LI × CD63 LI }\end{array}$ & $\begin{array}{r}-0.44 \\
-0.68 \\
0.01\end{array}$ & $\begin{array}{l}0.65 \\
0.51 \\
1.01\end{array}$ & $\begin{array}{l}0.008 \\
0.01 \\
0.04\end{array}$ \\
\hline Cancer-specific survival & $P=0.00001$ & $\begin{array}{l}\text { Age (years) } \\
\text { Complete surgery } \\
\text { Radiotherapy+temozolomide } \\
\text { TIMP-4 LI × CD63 LI }\end{array}$ & $\begin{array}{r}0.02 \\
-0.42 \\
-0.66 \\
0.01\end{array}$ & $\begin{array}{l}1.02 \\
0.66 \\
0.52 \\
1.01\end{array}$ & $\begin{array}{l}0.01 \\
0.02 \\
0.04 \\
0.02\end{array}$ \\
\hline
\end{tabular}

The Model/P-value indicates the overall level of significance of the model. Aside from 'age' and 'TIMP-4/CD63 co-expression' (continuous variables), all of the other variables are binary. These variables distinguish between complete and partial surgery and radiotherapy + temozolomide compared with radiotherapy alone. $\beta$ indicates the coefficient of each variable in the linear combination. $e^{\beta}$ value indicates that the risk of recurrence/death is increased by $e^{\beta}$ percentage for patients belonging to the indicated category (in the case of binary variables) or per year of patients' ages. A negative value means a positive impact on survival. The individual $P$-values are the levels of significance of the independent contributions of each variable to the model.

particularly in the glioblastoma subgroup. This work identifies, for the first time, the contribution of CD63 and TIMP-4 in this particular field of study.

To our knowledge, this study is based on the largest glioma series analyzed for TIMP-4 expression and is also the first to characterize CD63 expression in normal brain and in gliomas. Using in situ hybridization, Groft et $a l^{13}$ previously studied the cellular origin of TIMP-4 in some glioblastoma. As we observed here, they noted that TIMP-4 transcripts were mainly localized to malignant astrocytoma cells. ${ }^{13}$ Data regarding CD63 in gliomas were only provided in the work of Shirahata et $a l^{29}$ in which $C D 63$ appeared in a list of genes highly overexpressed in glioblastomas as compared with anaplastic oligodendrogliomas. Our study extends this result by detecting low CD63 expression levels in oligodendrogliomas of all grades compared with 


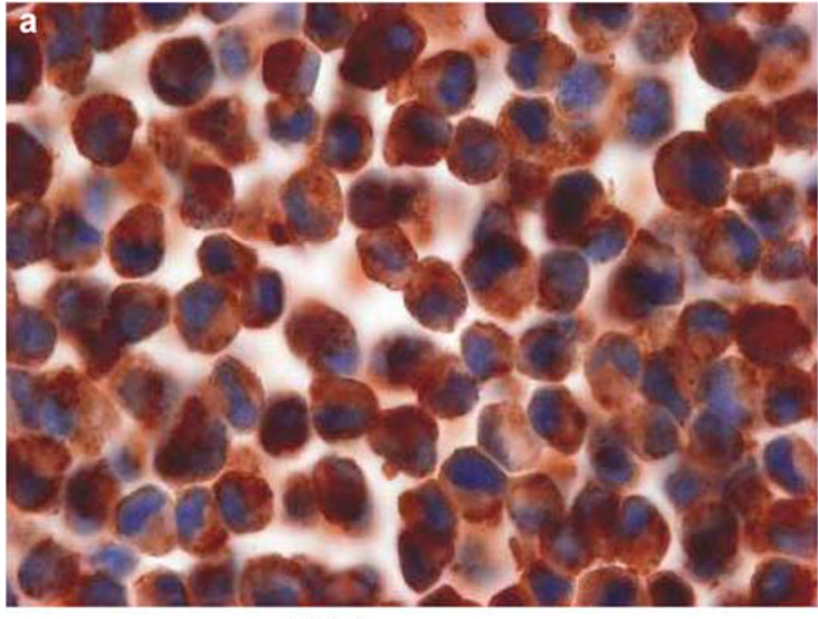

CD63

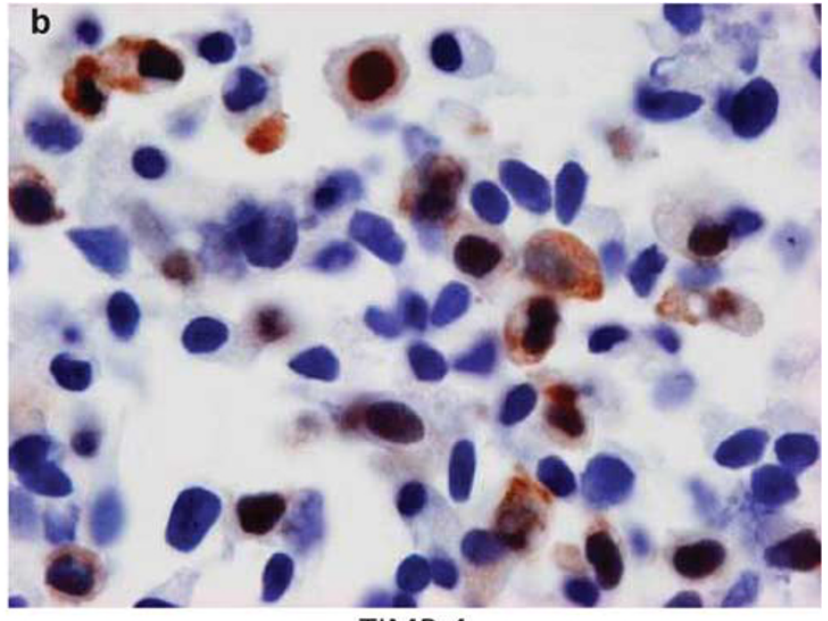

TIMP-4

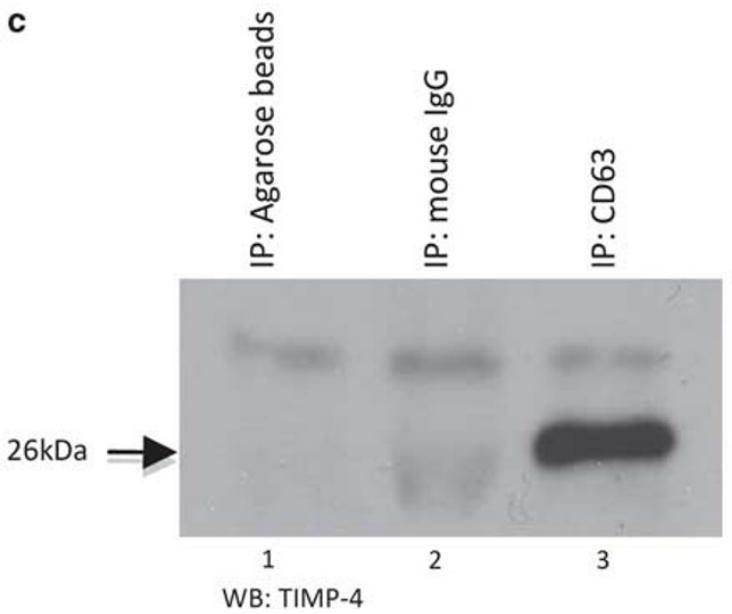

Figure 5 (a, b) Immunohistochemical (IHC) staining of (a) CD63 and (b) TIMP-4 in LN229 glioblastoma cells. (c) Immunoblotting (WB) using an anti TIMP-4 antibody showing TIMP-4 and CD63 binding in LN229 glioblastoma cells. Cell lysates were immunoprecipitated (IP) with an anti-CD63 antibody (lane 3), the corresponding IgG isotype (lane 2) or water (lane 1).

astrocytomas. This work confirms that TIMP-4 is overexpressed in pilocytic astrocytomas compared with diffuse grade II astrocytomas. In addition, we note that CD63 exhibits the same distribution in both tumor subgroups. Finally, we found that the best semi-quantitative scoring enabling to easily discriminate between both tumor subgroups corresponds to a CD63 $\mathrm{LI} \leq 10 \%$. Pilocytic astrocytomas and diffuse grade II astrocytomas exhibit characteristic morphological features allowing diagnosis of these tumors in most instances. ${ }^{2}$ However, morphological variety and limited material at examination could result in major difficulties in distinguishing pilocytic astrocytomas from grade II diffuse astrocytomas. ${ }^{30}$ This is especially true when only small specimens are available, as in the case of stereotactical biopsies. Clinical and neuroradiological features often facilitate diagnosis, but the data available are sometimes insufficient to distinguish between these two types of tumors. In addition, in the case of some pilocytic astrocytomas, the tumor cells infiltrate the surrounding normal parenchyma and, as seen under the microscope, may mimic the infiltrative pattern of diffuse astrocytomas. ${ }^{30,31} \mathrm{In}$ such cases, additional tumor-specific markers besides histological features would greatly assist in the diagnosis. CD63 was first discovered as an abundantly expressed surface antigen in early-stage melanoma cells. ${ }^{32}$ A negative relationship between CD63 expression and increased malignancy or invasiveness has been reported in many tumors, including ovarian, lung, breast and colon cancers, as well as in melanoma. ${ }^{23}$ Although these reports contradict our results on glioblastoma, they correlate with the high CD63 expression level noted in pilocytic astrocytoma, an indolent, noninvasive tumor. The high TIMP-4 and CD63 expressions noted here in both pilocytic astrocytomas and glioblastomas, which are radically different tumor subtypes regarding their biological behavior, are quite surprising. This finding might be related to a different biological implication of these biomarkers in pilocytic astrocytomas and glioblastomas, respectively. Interestingly, pilocytic astrocytomas strongly 
express both CD63 and TIMP-4, but none of these markers influence pilocytic astrocytoma patient outcomes. In contrast to pilocytic astrocytomas, high TIMP-4/CD63 co-expression in glioblastomas negatively impacts patients' survival. In addition, the reduced expression of both markers observed in the invasive part of glioblastomas compared with that in the tumor bulk suggests that these proteins may act preferentially in the biology of glioblastoma tumor bulk formation. These results thus agree with the multifaceted behavior of TIMP-4 previously reported in literature. Although different studies identified a negative impact of TIMP-4 in tumor cells invasion in vitro, ${ }^{13,33}$ others suggested that TIMP-4 has a signaling capacity distinct from the inhibitory activity of its matrix metalloproteinases that, like that of TIMP- $1,{ }^{19}$ can be initiated by binding to CD63. ${ }^{18}$ This concept is reinforced here by novel evidence for an association between TIMP-4 and CD63 in LN229 cells, a glioblastoma cell line. Of the signaling roles involved in tumor aggressiveness, anti-apoptotic functions are reported for both TIMP-1 and TIMP-4, which might share the same receptor. ${ }^{17-19}$ This concept remains to be validated regarding TIMP-4. CD63 was observed to interact with many different molecules, including TIMP-1, integrins and some Src kinases. ${ }^{19,34}$ Recently, Milano et $a l^{35}$ showed that a novel inhibitor of Src kinases improved the therapeutic efficacy of temozolomide, resulting in a significant increase in glioblastoma autophagic cell death. Interestingly, the growth-inhibitory effects of the same inhibitor in HMC-1 mast cells were found to be associated with a decrease in CD63 expression in tumor cells. ${ }^{36}$ To the best of our knowledge, this relationship has never been investigated in glioblastoma cells.

In conclusion, this work provides the first evidence of a TIMP-4/CD63 association in glioblastoma tumor cells. It identifies TIMP-4 and CD63 as markers of the astrocytic phenotype in patients with gliomas. In addition, this work highlights the independent contribution of high TIMP-4/CD63 co-expression to the adverse outcomes of patients with glioblastoma. These new findings regarding TIMP-4 and CD63 in human astrocytomas may open new avenues in the search for novel targets in the treatment of these tumors. Additional in vitro studies are still needed to better approach the biological involvement of both markers in astrocytoma carcinogenesis.

\section{Acknowledgements}

We thank Miss Andra Negulescu, Dina Milowic, Françoise Hulot and Delphyn Hastir for their help in patients' data collection. We thank Miss Jennifer Nadelmann for her redacting assistance. This work has been carried out with the support of a grant awarded by the 'Fonds Erasme' and the 'Fonds Yvonne Boël', Brussels, Belgium.

\section{Disclosure/conflict of interest}

The authors declare no conflict of interest.

\section{References}

1 Giese A, Bjerkvig $\mathrm{R}$, Berens $\mathrm{ME}$, et al. Cost of migration: invasion of malignant gliomas and implications for treatment. J Clin Oncol 2003;21:1624-1636.

2 Louis DN, Ohgaki H, Wiestler OD, et al. The WHO Classification of Tumors of the Central Nervous System, 3rd ed. International Agency for Research on Cancer: Lyon, 2007.

3 Stupp R, Mason WP, van den Bent MJ, et al. Radiotherapy plus concomitant and adjuvant temozolomide for glioblastoma. N Engl J Med 2005;352:987-996.

4 Fernandez C, Figarella-Branger D, Girard N, et al. Pilocytic astrocytomas in children: prognostic factors: a retrospective study of 80 cases. Neurosurgery 2003; 53:544-553.

5 Bondy ML, Scheurer ME, Malmer B, , et al., Brain Tumor Epidemiology Consortium. Brain tumor epidemiology: consensus from the Brain Tumor Epidemiology Consortium. Cancer 2008;113:1953-1968.

6 Sanson M, Marie Y, Paris S, et al. Isocitrate dehydrogenase 1 codon 132 mutation is an important prognostic biomarker in gliomas. J Clin Oncol 2009;27:4150-4154.

7 Forshew T, Tatevossian RG, Lawson AR, et al. Activation of the ERK/MAPK pathway: a signature genetic defect in posterior fossa pilocytic astrocytomas. J Pathol 2009;218:172-181.

8 Cairncross G, Berkey B, Shaw E, et al. Phase III trial of chemotherapy plus radiotherapy compared with radiotherapy alone for pure and mixed anaplastic oligodendroglioma: Intergroup Radiation Therapy Oncology Group Trial 9402. J Clin Oncol 2006;24:2707-2714.

9 Rorive S, Maris C, Debeir O, et al. Exploring the distinctive biological characteristics of pilocytic and low-grade diffuse astrocytomas using microarray gene expression profiles. J Neuropathol Exp Neurol 2006; 65:794-807.

10 Baker AH, Edwards DR, Murphy G. Metalloproteinase inhibitors: biological actions and therapeutic opportunities. J Cell Sci 2002;115:3719-3727.

11 Jiang Y, Goldberg ID, Shi YE. Complex roles of tissue inhibitors of metalloproteinases in cancer. Oncogene 2002;21:2245-2252.

12 Crocker SJ, Pagenstecher A, Campbell IL. The TIMPs tango with MMPs and more in the central nervous system. J Neurosci Res 2004;75:1-11.

13 Groft LL, Muzik H, Rewcastle NB, et al. Differential expression and localization of TIMP-1 and TIMP-4 in human gliomas. Br J Cancer 2001;85:55-63.

14 Melendez-Zajgla J, Del Pozo L, Ceballos G, et al. Tissue inhibitor of metalloproteinases-4. The road less traveled. Mol Cancer 2008;7:85.

15 Bister V, Skoog T, Virolainen S, et al. Increased expression of matrix metalloproteinases-21 and -26 and TIMP-4 in pancreatic adenocarcinoma. Mod Pathol 2007;20:1128-1140.

16 Lizarraga F, Espinosa M, Maldonado V, et al. Tissue inhibitor of metalloproteinases- 4 is expressed in cervical cancer patients. Anticancer Res 2005;25:623-627.

17 Jiang Y, Wang M, Celiker MY, et al. Stimulation of mammary tumorigenesis by systemic tissue inhibitor 
of matrix metalloproteinase 4 gene delivery. Cancer Res 2001;61:2365-2370.

18 Stetler-Stevenson WG. Tissue inhibitors of metalloproteinases in cell signaling: metalloproteinase-independent biological activities. Sci Signal 2008;1:re6.

19 Jung KK, Liu XW, Chirco R, et al. Identification of CD63 as a tissue inhibitor of metalloproteinase-1 interacting cell surface protein. EMBO J 2006;25:3934-3942.

20 Qi JH, Ebrahem Q, Moore N, et al. A novel function for tissue inhibitor of metalloproteinases-3 (TIMP3): inhibition of angiogenesis by blockage of VEGF binding to VEGF receptor-2. Nat Med 2003;9:407-415.

21 Seo DW, Li H, Guedez L, et al. TIMP-2 mediated inhibition of angiogenesis: an MMP-independent mechanism. Cell 2003;114:171-180.

22 Chirco R, Liu XW, Jung KK, et al. Novel functions of TIMPs in cell signaling. Cancer Metastasis Rev 2006; 25:99-113.

23 Pols MS, Klumperman J. Trafficking and function of the tetraspanin CD63. Exp Cell Res 2009;315:1584-1589.

24 Decaestecker C, Moles Lopez X, D'Haene $\mathrm{N}$, et al. Requirements for the valid quantification of immunostains on tissue microarray materials using image analysis. Proteomics 2009;9:4478-4494.

25 Rorive S, Berton A, D’Haene N, et al. Matrix metalloproteinase-9 interplays with the IGFBP2-IGFII complex to promote cell growth and motility in astrocytomas. Glia 2008;56:1679-1690.

26 D'Haene N, Maris C, Sandras F, et al. The differential expression of Galectin-1 and Galectin-3 in normal lymphoid tissue and non-Hodgkin's and Hodgkin's lymphomas. Int J Immunopathol Pharmacol 2005;18:431-443.

27 Gamboa-Dominguez A, Dominguez-Fonseca C, Quintanilla-Martinez L, et al. Epidermal growth factor receptor expression correlates with poor survival in gastric adenocarcinoma from Mexican patients: a multivariate analysis using a standardized immuno- histochemical detection system. Mod Pathol 2004; 17:579-587.

28 Flynn JR, Wang L, Gillespie DL, et al. Hypoxiaregulated protein expression, patient characteristics, and preoperative imaging as predictors of survival in adults with glioblastoma multiforme. Cancer 2008;113: 1032-1042.

29 Shirahata M, Iwao-Koizumi K, Saito S, et al. Gene expression-based molecular diagnostic system for malignant gliomas is superior to histological diagnosis. Clin Cancer Res 2007;13:7341-7356.

30 Giannini C, Scheithauer BW. Classification and grading of low-grade astrocytic tumors in children. Brain Pathol 1997;7:785-798.

31 Tihan T, Fisher PG, Kepner JL, et al. Pediatric astrocytomas with monomorphous pilomyxoid features and a less favorable outcome. J Neuropathol Exp Neurol 1999;58:1061-1068.

32 Hotta H, Ross AH, Huebner K, et al. Molecular cloning and characterization of an antigen associated with early stages of melanoma tumor progression. Cancer Res 1998;48:2955-2962.

33 Wang M, Liu YE, Greene J, et al. Inhibition of tumor growth and metastasis of human breast cancer cells transfected with tissue inhibitor of metalloproteinase 4. Oncogene 1997;14:2767-2774.

34 Iida J, Skubitz AP, McCarthy JB, et al. Protein kinase activity is associated with CD63 in melanoma cells. J Transl Med 2005;3:42.

35 Milano V, Piao Y, LaFortune T, et al. Dasatinib-induced autophagy is enhanced in combination with temozolomide in glioma. Mol Cancer Ther 2009;8:394-406.

36 Gleixner KV, Mayerhofer $\mathrm{M}$, Sonneck K, et al. Synergistic growth-inhibitory effects of two tyrosine kinase inhibitors, dasatinib and PKC412, on neoplastic mast cells expressing the D816V-mutated oncogenic variant of KIT. Haematologica 2007;92:1451-1459. 